\title{
The Relationship Between Magnesium Supplementation and Glycemic Control in Diabetic Patients: A Review
}

\author{
Doaa K. Ibrahim ${ }^{1}$, Zeina A. Al-Thanoon ${ }^{2}$ \\ College of pharmacy, University of Mosul, Mosul-Iraq. \\ Corresponding author: doaa.php3@ student.uomosul.edu.iq \\ Received $\quad \underline{\text { Accepted }}$ \\ 08-06-2021 29-07-2021
}

\begin{abstract}
Background: Diabetes mellitus is a chronic disease with increasing worldwide prevalence. There are many studies to observe the role of minerals on the glycemic state in diabetic patients. One of the in the human se minerals is magnesium. Magnesium is the fourth most abundant mineral in the human body. It has a role in the action of more than 300 enzymes most of them is ATP-dependent reactions. Finally, magnesium has an effect on glucose metabolism, lipid metabolism, nucleic acid synthesis, and energy production. The relationship between magnesium and DM is complex and multifactorial. There is an association between hypomagnesemia and insulin resistance by affecting phosphorylation of insulin receptors, insulin signaling and insulin action. Another consequence of hypomagnesemia is oxidative stress which is also present in the pathogenesis of DM. Objective: This work is trying to emphasize the important role of magnesium in the control of the glycemic state in diabetic patients through highlighting the studies conducted to correlate between magnesium level in the body, dietary magnesium intake, or magnesium supplementation with risk of diabetes mellitus occurrence, insulin sensitivity or glycemic control in diabetic patients. This article suggested that magnesium therapy may be beneficial in improving the clinical parameters of diabetic patients including $\mathrm{HbA} 1 \mathrm{c} \%$, fasting serum glucose, and fasting insulin level.
\end{abstract}

Keywords: Magnesium, Diabetes mellitus, Glycemic control, Insulin resistance.

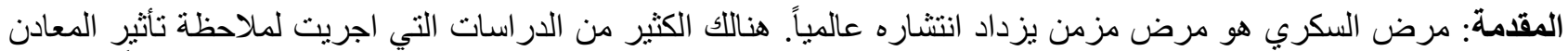

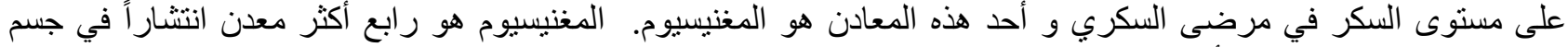

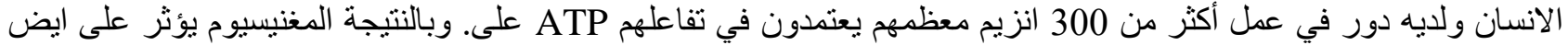

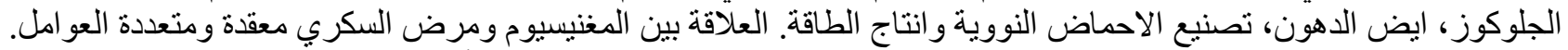

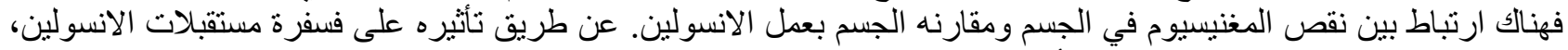

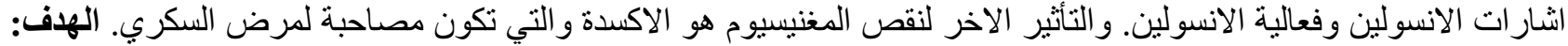

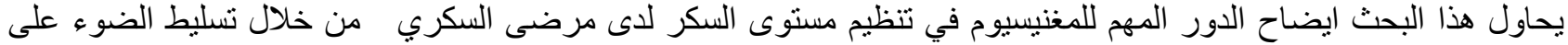

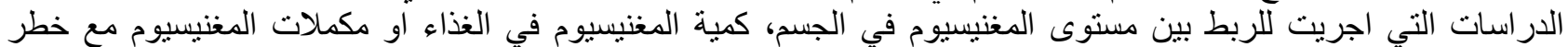

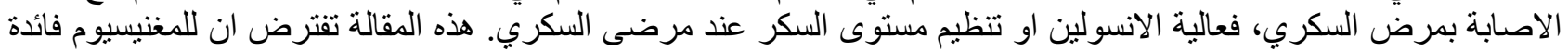

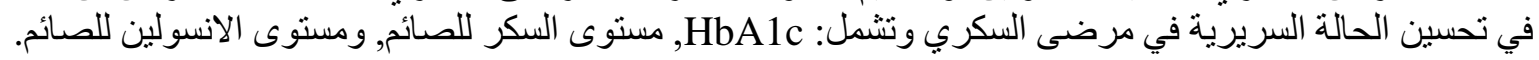

الكلمات المفتاحية: المغنيسيوم، مرض السكري، تنظيم مستوى السكر، مقاومة الانسولين. 


\section{Introduction}

$\mathrm{M}$ agnesium is the eighth abundant element in the earth while in the human body is the fourth most common mineral and in the cells is the most common mineral abundance after potassium in the abundance. Magnesium has an important role in many physiological functions, also it used clinically (1)

The relevant physical property of magnesium is hydrated with stronger bonds than that of water with other cations so its dehydration is also difficult. This property explains many features of magnesium such as calcium antagonism by magnesium as calcium pass the biological membrane more readily than magnesium because hydration of magnesium make it larger ${ }^{(1)}$ Another correlation between magnesium and calcium is competition for plasma protein- binding sites and magnesium act as an inhibitor of inositol triphosphate (IP-3) gated calcium channel $^{(2)}$. There is a study that observed this antagonism by measurement of serum calcium and serum magnesium through a period of magnesium treatment that found a reduction in calcium level with elevation in magnesium level ${ }^{(3)}$.

Magnesium distribution in the human body is as follows $99 \%$ of body magnesium is located in an intracellular compartment (bone, muscles and non-muscular soft tissue) About $50-60 \%$ of this percent present in bone. About $1 \%$ of total body magnesium resides in the blood (red blood cells and serum). In serum it is either presented as free ionized, protein bound or anions complexes. The biologically important part is the free ionized magnesium $\left(\mathrm{Mg}^{2+}\right)$. Magnesium is exchanged between extracellular compartment and skeletal muscle to maintain normal serum magnesium concentration ${ }^{(1)}$.

Magnesium is a cofactor in greater than 300 enzymatic reactions. Most of them are ATP-dependent reactions. These reactions include glucose metabolism, lipid metabolism, nucleic acid synthesis, and muscle contraction ${ }^{(2)}$.

The recommended daily allowance of magnesium is between $350-420 \mathrm{mg}$ in adult males and $280-360 \mathrm{mg}$ in the adult females. The main dietary sources of magnesium are green vegetables and fruits, nuts, whole grains, legumes and meat ${ }^{(\mathbf{1})}$ Also, water is another source of magnesium. Processing and boiling of food reduce its magnesium content. Nowadays, the processed food is commonly consumed in our daily routine $\operatorname{diet}^{(2,4)}$.

\section{Magnesium absorption and excretion}

Magnesium is absorbed in the human body through two pathways. Firstly, passive paracellular pathway in the small intestine. Secondly, active transcellular pathway in the large intestine. The major mechanism of absorption is the paracellular pathway. The extent of intestinal magnesium absorption is not constant that is ranging from one quarter to three quarters of consumed magnesium depending on many factors including serum magnesium level, age of individual, concurrent food composition and others ${ }^{(1,5)}$.

The kidney is the main body organ responsible for the excretion of excess body magnesium as free serum magnesium is filtered by the glomerulus and the extent of renal tubular reabsorption will determine the urinary magnesium concentration ${ }^{(1,5)}$.

Magnesium homeostasis is also regulated by hormones such as parathyroid hormones, vitamin $\mathrm{D}$ and estrogen ${ }^{(4)}$. 


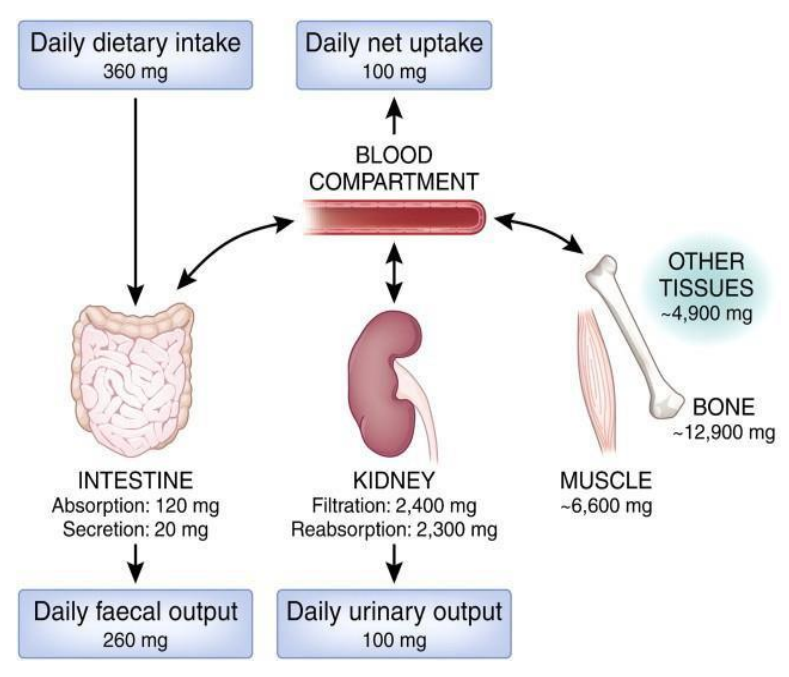

Figure (1): Magnesium balance inhuman $\operatorname{body}^{(1)}$

\section{Estimation of magnesium status in the body:}

\section{Serum magnesium measurement}

The $\mathrm{Mg}^{2+}$ concentration in serum does not necessarily reflect the overall level of magnesium in the body as only about $0.3 \%$ of total body magnesium content is present in serum. But this is the most suitable and common measurement of body magnesium status $^{(4)}$.

\section{Urinary magnesium excretion assessment}

To measure urinary magnesium excretion, it is necessary to Collect a 24hour urine sample. Usually, this test is performed to evaluate renal excretion and intestinal absorption of magnesium as values higher than normal level of urinary magnesium is indicative of renal wasting of magnesium while values lower than normal level is indicative of reduced intestinal absorption of magnesium $^{(1)}$.

\section{Physiological functions of magnesium}

The physiological functions of magnesium are diverse and multiple. The biological role of magnesium is mainly contributing to the chelating of it with ATP forming Mg-ATP complex acting as a cofactor in more than 300 enzymatic reactions especially kinase system. Magnesium has an effect on lipid metabolism, protein synthesis, nucleic acid synthesis, ion channel regulation, energy production and hormone receptor interaction. Magnesium also has antioxidant properties $^{(6)}$.

Magnesium deficiency is associated with increased production of free radicals, inhibition of antioxidant enzymes, inhibition of nitric oxide release, lipid peroxidation and higher level of malonaldehyde ${ }^{(7)}$.

Magnesium has a role in lipid metabolism and the mechanism is as follows: 3-hydroxy 3-methyl glutaryl Co A reduction is the rate limiting step in cholesterol biosynthesis. This step requires hydroxy methyl glutaryl Co A reductase (HMG-Co A reductase) enzyme to be accomplished. This enzyme is inactivated by a mechanism involve phosphorylation of the enzyme by reaction in the presence of $\mathrm{Mg}$ ATP complex by this way magnesium control the function of HMG-Co A reductase, and finally the biosynthesis of cholesterol $^{(8)}$.

Other role of magnesium in lipid metabolism is observed by the effects of magnesium deficiency in increasing efflux of free fatty acids form cells, increasing triglyceride synthesis and stimulation of VLDL (Very Low Density Lipoprotein) production $^{(9)}$.

Magnesium is also expected to have a helpful effect in controlling blood pressure by antagonism of calcium and reduction of catecholamine release with subsequent reduction in peripheral resistance ${ }^{(10)}$.

Additionally, Magnesium stimulates nitric oxide (NO) and prostaglandin I2 release which act as vasodilator. All these factors collectively result in the reduction of blood pressure by magnesium ${ }^{(11)}$. 
The correlation between magnesium and glucose hemostasis is manifold including its vital role in glycolysis, its effect on insulin secretion and action ${ }^{(12)}$.

\section{Hypomagnesemia}

It means serum magnesium concentration lower than $0.61 \mathrm{mmole} / \mathrm{l}(1,5$ $\mathrm{mg} / \mathrm{dl})^{(13)}$. There are many causes of hypomagnesemia including: reduction in magnesium intake, reduction in magnesium absorption, and increase in magnesium excretion. In addition, it may result from diseases such as diabetes mellitus, cirrhosis, hyperthyroidism, and hypercalcemia. Medicines also can cause magnesium deficiency such as diuretics, digoxin, and proton pump inhibitor ${ }^{(\mathbf{1})}$.

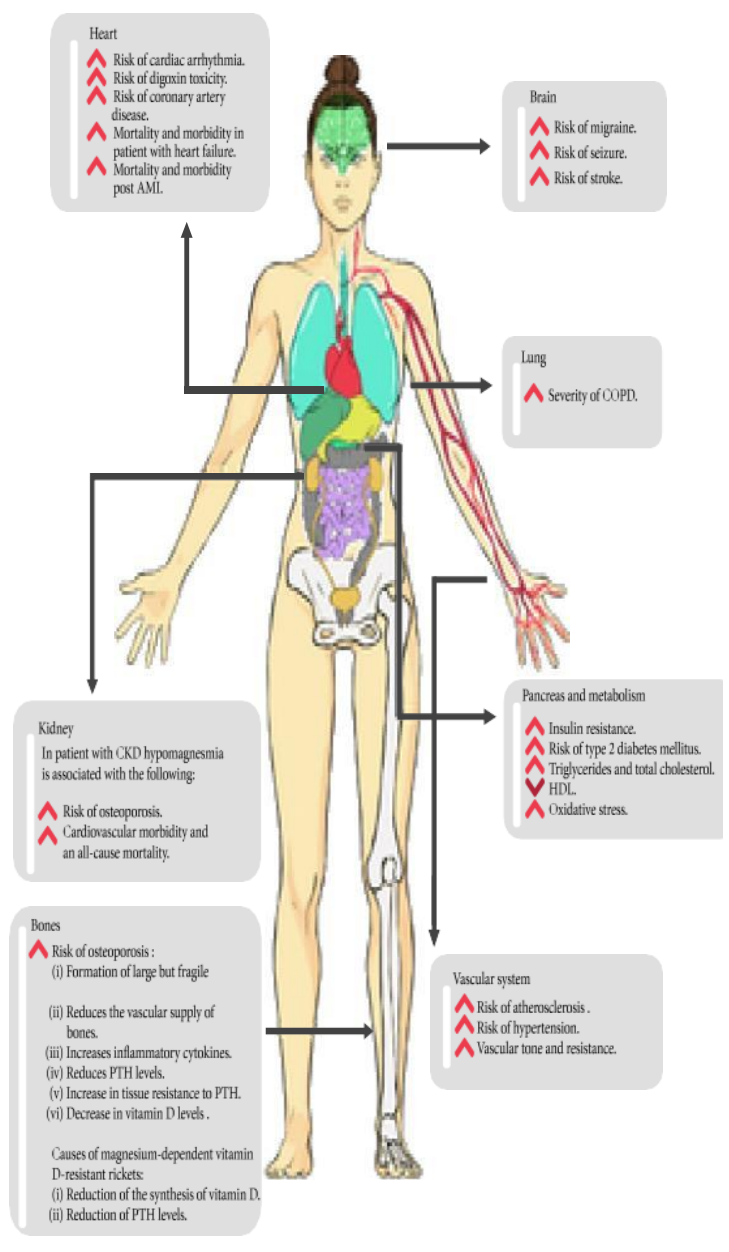

Figure (2): The impact of hypomagnesaemia (4)

\section{Diabetes mellitus (DM)}

Diabetes mellitus is a chronic disease with increasing worldwide prevalence. 90$95 \%$ of diabetic patients are type 2 DM. In DM the main complaint is hyperglycemia with reduced insulin action or secretion that is associated with many factors (genetic, environmental and medical risk factors). DM has micro- and macro- vascular complications $^{(14)}$.

In Iraq, the prevalence of type $2 \mathrm{DM}$ in 2015 was about four patients in every 100 Iraqi individuals ${ }^{(15)}$.

The etiology of type $2 \mathrm{DM}$ is associated with many factors but it is mainly caused by reduction in insulin sensitivity with an increase in the pancreatic secretion of insulin but the ability of pancreatic $\beta$ cells to compensate this impairment in insulin action decreases with time resulting in a lower level of insulin secretion ${ }^{(16,17)}$.

The main goal of the management of $\mathrm{DM}$ is to maintain normal serum glucose levels and to prevent long term complications of $\mathrm{DM}^{(18)}$. The plan of management of type $2 \mathrm{DM}$ requires lifestyle modification with the reduction in dietary carbohydrates and fat intake, prolongation of exercise time and weight reduction. Moreover, pharmacologic intervention is required in diabetic patients. There are many groups of anti-diabetic agents and insulin therapy ${ }^{(16)}$.

\section{The prevalence of hypomagnesaemia in diabetic patients}

The prevalence of magnesium deficiency in patients with DM is high due to several reasons. One of them is that the pathophysiology of this disease increases magnesium excretion by hyperinsulinemia, hyperglycemia and glycosuria all of these 
factors reduces renal tubular reabsorption and subsequently increases magnesium $\operatorname{loss}^{(19)}$. Hyperinsulinemia also results in hypomagnesemia by increasing cellular uptake of magnesium with reducing the serum concentration of magnesium ${ }^{(4,13)}$.

This high incidence of hypomagnesaemia in DM has observed in many studies one of them is a cross sectional study conducted in India on 250 diabetic patients found that the prevalence of hypomagnesemia is $44 \% \%^{(20)}$.

Other studies in Iraq conducted in Kirkuk found that the level of serum magnesium in diabetic patients $(0.6$ mmole/l) is lower significantly than that in healthy individuals $(1.07 \mathrm{mmole} / \mathrm{l})^{(21)}$.

\section{The association between magnesium deficiency and type 2 diabetes}

The relationship between magnesium and DM is complex and multifactorial. Magnesium is involved in the phosphorylation of tyrosine kinase insulin receptor, the insulin signaling regulation. Itis also affecting insulin action on cellular uptake of glucose by insulin sensitive glucose transporter (GLUT 4) and affecting glucose stimulated insulin secretion ${ }^{(22)}$.

Other causes of insulin resistance in hypomagnesaemia are associated with increased calcium influx to adipocyte with subsequent oxidative stress $^{(4)}$. Finally, magnesium deficiency is associated with worsening insulin sensitivity as shown in Figure (2) ${ }^{(23)}$.

Another link between magnesium deficiency and DM is the oxidative stress with enhanced free radical production that is found in both cases $^{(13)}$.

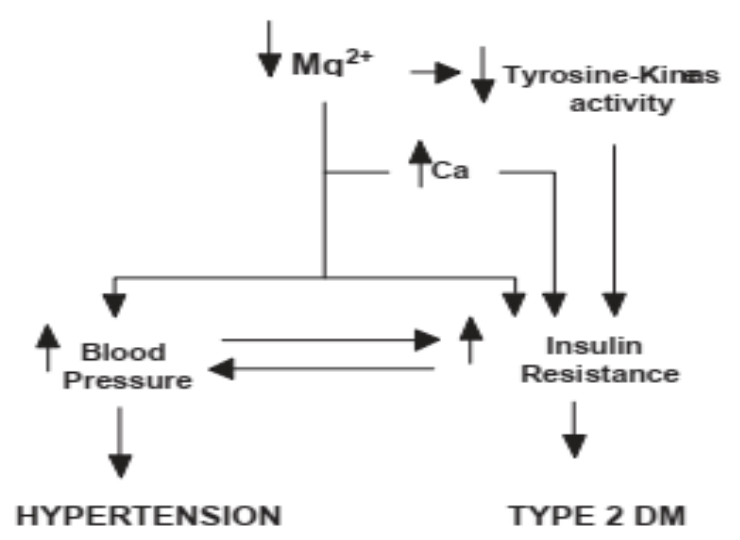

Figure (3): The relation between $\mathrm{Mg}$ deficiency with type 2 DM and hypertension $^{(24)}$

\section{The relation between magnesium intake and incidence of DM}

Over the last century, there are many studies have been conducted to evaluate the effect of hypomagnesaemia on DM.

An animal study of magnesium effects on insulin resistance is performed by induction of $\mathrm{DM}$ in rats and initiating magnesium supply to them. This study found an improvement in insulin resistance by stimulating insulin receptor expression and increasing the binding constant and affinity of insulin receptors ${ }^{(25)}$. This is in agree with other studies on diabetic rats conducted by (Sohrabipour et al., 2018) found an improvement in insulin sensitivity with magnesium supplementation ${ }^{(26)}$.

Several epidemiological studies were performed on humans to observe the effect of magnesium intake on the incidence of DM. A meta -analysis of 13 cohort study found that there is an inverse relationship between them that the degree of correlation depend on the dose of magnesium ${ }^{(27)}$

Another study with similar results is a meta-analysis of seven cohort studies that found a $15 \%$ reduction in diabetes risk witha $100 \mathrm{mg}$ increase in daily magnesium intake $^{(28)}$.

61616161 
This is also observed by a meta- analysis of 25 cohort studies performed by (Fang, $2016)^{(29)}$ and a meta-analysis of 11 cohort studies conducted by (Wu et al.,2017) correlate serum magnesium level with the

incidence of DM that found lower magnesium level is associated with a higher incidence rate of $\mathrm{DM}^{(\mathbf{3 0})}$.

Other study performed by the Nurses' Health Study (NHS) and Health Professionals' Follow-up Study (HFS), on 127,932 research participants $(85,060$ female and 42,872 male) with 18 years follow up of female participants and 12 years follow up of male participants. This study concluded increase in the risk of developing DM in participants with lower amount of magnesium intake ${ }^{(24)}$.

\section{Studies emphasize the improvement in insulin resistance by magnesium supplements}

There are several studies performed to evaluate magnesium effects on insulin resistance. One of these studies is a double blind clinical trials in Mexico used magnesium chloride supplements for 4 months found a significant improvement in glycemic status as HbA1c percent and fasting glucose level are reduced significantly, and fasting insulin level is increased while HOMA-IR (Homeostatic model assessment for insulin resistance) is decreased $^{(31)}$. (HOMA-IR is a convenient method to estimate insulin resistance. It is calculated by multiplying fasting plasma insulin (FPI) with fasting plasma glucose (FPG), then dividing by the constant $\left.22.5^{(32)}\right)$.

\section{HOMA-IR= $($ FPI $\times$ FPG $) / 22.5$}

A systematic review of 21 randomized clinical trials found that magnesium supplementation for period 4-16 weeks is associated with detectable change in HOMA-IR index either the participants are diabetic or not ${ }^{(33)}$.
This is similar to results of metaanalysis performed by (Hruby et al., 2013) on 15 cohort study observed a significant negative correlation between intake of dietary magnesium and insulin level $(\mathrm{p}<$ $0.0001)^{(34)}$.

\section{Studies approve ineffectiveness of magnesium on insulin resistance}

A randomized clinical trial performed on 54 type 2 diabetic patients divided into two groups first group receive magnesium supplements for 12 weeks (magnesium supplement group) and second group is a control group. It found an insignificant change in magnesium supplement group of the following parameters: HOMA-IR index, fasting plasma insulin level and $\mathrm{HbA} 1 \mathrm{c} \%$ while there is a significant reduction in fasting blood glucose level ${ }^{(35)}$. Other study with similar results is the study found that use of $400 \mathrm{mg}$ magnesium supplements for three months did not significantly improve insulin resistance which detected by measurement of HOMA -IR index $(\mathrm{p}=0.36)$ and insulin level $(\mathrm{mU} / \mathrm{L})$ with $(\mathrm{p}=0.24)$ and glycemic control by measurement of fasting glucose $^{(36)}$.

\section{Studies emphasize the improvement in glycemic parameters by magnesium}

Many studies performed to observe the effects of dietary magnesium intake, serum magnesium level or magnesium supplements on glycemic control in diabetic patients. Here we will mention several examples of them. Firstly, a clinical trial conducted to demonstrate the effect of magnesium supplements on fasting glucose in prediabetics with hypomagnesaemia found a significant reduction in fasting glucose $(\mathrm{p}=$ 0.004) While fasting insulin level $(\mathrm{p}=0.31)$ and HOMA-IR index $(\mathrm{p}=0.04)$ does not improve significantly that fasting insulin ${ }^{(37)}$.

Secondly, a study performed in Egypt showed the correlation between low serum 
magnesium level with high HbA1c \% (p < $0.001)$ in children with type $1 \mathrm{DM}^{(38)}$.

The similar results of positive effects of magnesium supplements on glycemic parameters are also observed by study conducted by (Labban, 2019) ${ }^{(39)}$ and are observed by (Toprak et al., 2017) ${ }^{(40)}$.

There is also a survey made by interviewing 1573 non diabetics participants was done in Mexico which is found an inverse correlation between dietary magnesium intake and many parameters one of them is serum glucose ${ }^{(41)}$.

The relation of serum magnesium level on glycemic state have been studied by a study conducted in India found an inverse correlation between $\mathrm{HbAlc} \%$ in diabetic patients with serum magnesium level ${ }^{(42)}$.

Lastly, a systematic review of 12 placebo controlled clinical trials found that magnesium has benefits on glycemic indicators in diabetic patients with hypomagnesaemia but not in those with normomagnesemia. The trials included in the review use different doses, forms and duration of magnesium supplements ${ }^{(43)}$.

\section{Studies shows insignificant effects of magnesium supplements on glycemic control}

The previously mentioned studies are in controversy with other studies. A crosssectional study compare serum magnesium level with fasting serum glucose in 90 diabetic or hypertensive patients found insignificant relationship between them $(\mathrm{p}=$ $0.34)^{(44)}$.

A meta-analysis of 28 study found insignificant beneficial effects of magnesium on $\mathrm{HbA1c} \%$ which may be associated with short duration of supplementation $^{(45)}$.

In Iraq, at Azadi teaching hospital in Duhok a cross sectional study on 100 diabetic patients found no correlation between serum magnesium level and HA1c percent. Although this study has a limitation of small size of sample and it is not a clinical trial $^{(46)}$.

A clinical trial conducted on type 2 diabetic patients with normal serum magnesium level found no improvement in glycemic control but this study has a limitation of small sample size only 26 patients take magnesium supplements through the study period ${ }^{(18)}$.

\section{Conclusion}

According to the results of mentioned studies, there are controversy in the relation of low dietary ingestion or serum level of magnesium to the glycemic control in diabetic patients ${ }^{(47)}$.

Many studies on diabetic patients were performed to observe the interference between glycemic control and serum magnesium level. The clinical studies found a positive correlation of magnesium deficiency with risk of diabetes in prediabetics and negative correlation with glycemic control in diabetic patients.

The outcome of magnesium supplements on metabolic control in diabetic patients are related to the dose strength of magnesium supplements, the duration of magnesium supplementation those will determine the degree of improvement in glycemic control in diabetic patients.

Finally, magnesium supplements will improve the glycemic control in diabetic patients even this is not statistically significant.

More studies are required to determine the most effective dose and duration of magnesium treatment in diabetic patients.

Conflict of interest: There is no conflict of interest.

\section{References}

1. Jahnen-Dechent W, Ketteler M. 
Magnesium basics. Clinical kidney journal. 2012;5(Suppl_1):i3-i14.

2. Chaudhary DP, Sharma R, Bansal DD. Implications of magnesium deficiency in type 2 diabetes: a review. Biological trace element research. 2010;134(2):119-29.

3. ELDerawi WA, Naser IA, Taleb $\mathrm{MH}$, Abutair AS. The effects of oral magnesium supplementation on glycemic response among type 2 diabetes patients. Nutrients. 2019;11(1):44.

4. Al Alawi AM, Majoni SW, Falhammar H. Magnesium and human health: perspectives and research directions. International journal of endocrinology. 2018;2018.

5. Philipp Schuchardt J, Hahn A. Intestinal absorption and factors influencing bioavailability of magnesium-an update. Current Nutrition \& Food Science. 2017;13(4):260-78.

6. Veronese N, Demurtas J, Pesolillo G, Celotto S, Barnini T, Calusi $G$, et al. Magnesium and health outcomes: An umbrella review of systematic reviews and meta-analyses of observational and intervention studies. European journal of nutrition. 2020;59(1):263-72.

7. Morais JBS, Severo JS, Dos Santos LR, de Sousa Melo SR, de Oliveira Santos $\mathrm{R}$, de Oliveira ARS, et al. Role of magnesium in oxidative stress in individuals with obesity. Biological trace element research. 2017;176(1):20-6.

8. Rosanoff A, Seelig MS. Comparison of mechanism and functional effects of magnesium and statin pharmaceuticals. Journal of the American College of Nutrition. 2004;23(5):501S-5S.

9. Sajjan N, Shamsuddin M. A study of serum magnesium and dyslipidemia in type 2 diabetes mellitus patients. International Journal of Clinical Biochemistry and Research. 2016;3(1):36-41.

10. Dibaba DT, Xun P, Song Y, Rosanoff A, Shechter M, He K. The effect of magnesium supplementation on blood pressure in individuals with insulin resistance, prediabetes, or noncommunicable chronic diseases: a meta-analysis of randomized controlled trials. The American journal of clinical nutrition. 2017;106(3):921-9.

11. Asbaghi O, Hosseini R, Boozari B, Ghaedi E, Kashkooli S, Moradi S. The effects of magnesium supplementation on blood pressure and obesity measure among type 2 diabetes patient: a systematic review and meta-analysis of randomized controlled trials. Biological trace element research. 2020:1-12.

12. Glasdam S-M, Glasdam S, Peters $\mathrm{GH}$. The importance of magnesium in the human body: a systematic literature review. Advances in clinical chemistry. 2016;73:169-93.

13. Barbagallo $\mathrm{M}$, Dominguez LJ. Magnesium and type 2 diabetes. World journal of diabetes. 2015;6(10):1152.

14. Rasheed H, Elahi S, Ajaz H. Serum magnesium and atherogenic lipid fractions in type II diabetic patients of Lahore, Pakistan. Biological trace element research. 2012;148(2):165-9.

15. Mansour AA, Alibrahim NT, Alidrisi HA, Alhamza AH, Almomin AM, Zaboon IA, et al. Prevalence and correlation of glycemic control achievement in patients with type 2 diabetes in Iraq: A retrospective analysis of a tertiary care database over a 9year period. Diabetes \& Metabolic Syndrome: Clinical Research \& Reviews. 2020;14(3):265-72.

16. Chung IM, Rajakumar G, Subramanian U, Venkidasamy B, Khanna VG, Thiruvengadam M. Insights on the current status and advancement of diabetes mellitus type 2 and to avert complications: An overview. Biotechnology and applied biochemistry. 2020;67(6):920-8.

17. Javeed N, Matveyenko AV.Circadian etiology of type 2 diabetes mellitus. Physiology. 2018;33(2):138-50.

18. Navarrete-Cortes A, Ble-Castillo JL, 
Guerrero-Romero F, Cordova-Uscanga R, Juárez-Rojop IE, Aguilar-Mariscal H, et al. No effect of magnesium supplementation on metabolic control and insulin sensitivity in type 2 diabetic patients with normomagnesemia. Magnesium research. 2014;27(2):48-56.

19. Schwalfenberg GK, Genuis SJ. The importance of magnesium in clinical healthcare. Scientifica. 2017;2017.

20. Kumar P, Bhargava S, Agarwal PK, Garg A, Khosla A. Association of serum magnesium with type 2 diabetes mellitus and diabetic retinopathy. Journal of family medicine and primary care. 2019;8(5):1671.

21. Ahmed AS, Tahir PH. Physiological effects of some trace elements on some blood parameters in diabetic patients and their correlation with HbA1c. EurAsian Journal of BioSciences. 2020;14:7477-80.

22. Song Y, Dai Q, He K. Magnesium intake, insulin resistance, and type 2 diabetes. N Am J Med Sci. 2013;6:9-15.

23. Hadjistavri LS, Sarafidis PA, Georgianos PI, Tziolas IM, Aroditis CP, Hitoglou-Makedou A, et al. Beneficial effects of oral magnesium supplementation on insulin sensitivity and serum lipid profile. Medical Science Monitor. 2010;16(6):CR0CR.

24. Lopez-Ridaura R, Willett WC, Rimm EB, Liu S, Stampfer MJ, Manson JE, et al. Magnesium intake and risk of type 2 diabetes in men and women. Diabetes care. 2004;27(1):134-40.

25. Liu H, Li N, Jin M, Miao X, Zhang $\mathrm{X}$, Zhong W. Magnesium supplementation enhances insulin sensitivity and decreases insulin resistance in diabetic rats. Iranian Journal of Basic Medical Sciences. 2020;23(8):990.

26. Sohrabipour S, Sharifi MR, Sharifi M, Talebi A, Soltani N. Effect of magnesium sulfate administration to improve insulin resistance in type 2 diabetes animal model: using the hyperinsulinemic- euglycemic clamp technique. Fundamental \& clinical pharmacology. 2018;32(6):603- 16.

27. Dong J-Y, Xun P, He K, Qin L-Q. Magnesium intake and risk of type 2 diabetes: meta-analysis of prospective cohort studies. Diabetes care. 2011;34(9):2116-22.

28. Larsson S, Wolk A. Magnesium intake and risk of type 2 diabetes: a metaanalysis. Journal of internal medicine. 2007;262(2):208-14.

29. Fang X, Han H, Li M, Liang C, Fan $\mathrm{Z}$, Aaseth $\mathrm{J}$, et al. Dose-response relationship between dietary magnesium intake and risk of type 2 diabetes mellitus: a systematic review and meta-regression analysis of prospective cohort studies. Nutrients. 2016;8(11):739.

30. Wu J, Xun P, Tang Q, Cai W, He K. Circulating magnesium levels and incidence of coronary heart diseases, hypertension, and type 2 diabetes mellitus: a meta-analysis of prospective cohort studies. Nutrition journal. 2017;16(1):1-13.

31. Rodríguez-Morán M, GuerreroRomero F. Oral magnesium supplementation improves insulin sensitivity and metabolic control in type 2 diabetic subjects: a randomized double-blind controlled trial. Diabetes care. 2003;26(4):1147-52.

32. Qu H-Q, Li Q, Rentfro AR, FisherHoch SP, McCormick JB. The definition of insulin resistance using HOMA-IR for Americans of Mexican descent using machine learning. PloS one. 2011;6(6):e21041.

33. Simental-Mendia LE, Sahebkar A, Rodriguez-Moran M, Guerrero-Romero F. A systematic review and meta-analysis of

randomized controlled trials on the effects of magnesium supplementation on insulin sensitivity and glucose control. Pharmacological research. 2016;111:272-82.

34. Hruby A, Ngwa JS, Renström F, Wojczynski MK, Ganna A, Hallmans G, et al. Higher magnesium intake is associated 
with lower fasting glucose and insulin, with no evidence of interaction with select genetic loci, in a meta-analysis of 15 CHARGE Consortium Studies. The Journal of nutrition. 2013;143(3):345-53.

35. Solati M, Ouspid E, Hosseini S, Soltani N, Keshavarz M, Dehghani M. Oral magnesium supplementation in type II diabetic patients. Medical journal of the Islamic Republic of Iran. 2014;28:67.

36. de Souza MdLL. Magnesium replacement does not improve insulin resistance in patients with metabolic syndrome: a 12-week randomized doubleblind study. Journal of clinical medicine research. 2014;6(6):456.

37. Guerrero-Romero F, SimentalMendía L, Hernández-Ronquillo G, Rodriguez-Morán M. Oral magnesium supplementation improves glycaemic status in subjects with prediabetes and hypomagnesaemia: a double-blind placebocontrolled randomized trial. Diabetes \& metabolism. 2015;41(3):202-7.

38. Shahbah D, El Naga AA, Hassan T, Zakaria M, Beshir M, Al Morshedy S, et al. Status of serum magnesium in Egyptian children with type 1 diabetes and its correlation to glycemic control and lipid profile. Medicine. 2016;95(47).

39. Labban L, Thallaj N. The Effect of Magnesium Supplementation on Hba1c Level and Lipid Profile Among Type 2 Diabetics. Acta Scientifci Nutritional Health. 2019;3:07-12.

40. Toprak O, Kurt H, Sarı Y, Şarkış C, Us H, Kirik A. Magnesium replacement improves the metabolic profile in obese and pre-diabetic patients with mild-to-moderate chronic kidney disease: a 3-month, randomised, double-blind, placebocontrolled study. Kidney and Blood PressureResearch. 2017;42(1):33-42.

41. Castellanos-Gutiérrez A, SánchezPimienta TG, Carriquiry A, da Costa TH, Ariza AC. Higher dietary magnesium intake is associated with lower body mass index, waist circumference and serum glucose in Mexican adults. Nutrition journal. 2018;17(1):1-8.

42. Ramadass S, Basu S, Srinivasan A. SERUM magnesium levels as an indicator of status of Diabetes Mellitus type 2. Diabetes \& Metabolic Syndrome: Clinical Research \& Reviews. 2015;9(1):42-5.

43. Morais JBS, Severo JS, de Alencar GRR, de Oliveira ARS, Cruz KJC, do Nascimento Marreiro D, et al. Effect of magnesium supplementation on insulin resistance in humans: a systematic review. Nutrition. 2017;38:54-60.

44. Kaveh P, Tehrani AM, Mardani S. Association of serum magnesium level with resistant hyperlipidemia in diabetic and hypertensive patients. Journal of Nephropharmacology. 2019;9(1):e07-e.

45. Verma H, Garg R. Effect of magnesium supplementation on type 2 diabetes associated cardiovascular risk factors: a systematic review and metaanalysis. Journal of human nutrition and dietetics. 2017;30(5):621-33.

46. Saeed H, Haj S, Qasim B. Estimation of magnesium level in type 2 diabetes mellitus and its correlation with HbA1c level. Endocrinology, diabetes \& metabolism. 2019;2(1):e0048.

47. Sales CH, Pedrosa LdFC. Magnesium and diabetes mellitus: their relation. Clinical nutrition. 2006;25(4):55462. 